\title{
Stabilizing Predictors for Weakly Unstable Correctors
}

\author{
By Hans J. Stetter
}

1. Introduction. It is well known that Milne-Simpson's method

$$
y_{n+2}=y_{n}+\frac{h}{3}\left(f_{n}+4 f_{n+1}+f_{n+2}\right)
$$

should not be used for the numerical integration of $y^{\prime}=f(x, y)$ if $f_{v}<0$ along the true solution $y(x)$ although the solution of $(1)$ converges to $y(x)$ for fixed finite $x$ as $h \rightarrow 0$ (see, e.g., [1]). In fact, rapid oscillations, with an amplitude increasing exponentially as the numerical integration proceeds, will supersede the values approximating $y(x)$ and eventually destroy the meaningfulness of the computation. This "weak unstability" occurring with (1) and similar algorithms has been well I analyzed (e.g., [1, p. $248 \mathrm{ff}$.$] ) and procedures have been suggested to weaken its$ effect (e.g., [2]). We will show in this paper that it is quite easy to completely eliminate its cause: The combination of a judiciously chosen predictor with the weakly unstable corrector constitutes a strongly stable algorithm if the corrector is not iterated.

2. Analysis. Consider the $k$-step scheme

$$
\rho(E) y_{n}-h \sigma(E) f_{n}=0,
$$

where $\rho(z):=\sum_{\nu=0}^{k} \alpha_{v} z^{\nu}, \alpha_{k}=1 ; \sigma(z):=\sum_{\nu=0}^{k} \beta_{v} z^{\nu} ; E y_{n}:=y_{n+1} ; f_{n}:=f\left(x_{n}, y_{n}\right)$.

(2) is called D-stable or stable for $h \rightarrow 0$ if all zeros of $\rho$ are in $|z| \leqq 1$ and no multiple zeros are on $|z|=1$. (2) is of order $p$ if, for a sufficiently differentiable function $y$,

$$
\rho\left(E_{h}\right) y(x)-h \sigma\left(E_{h}\right) y^{\prime}(x)=O\left(h^{p+1}\right),
$$

where $E_{h} y(x):=y(x+h)$.

It is well known (e.g., [1]) that the sequence $y_{n}$ generated by a D-stable scheme (2) of order $p \geqq 1$ converges in an obvious sense to the solution $y(x)$ of $y^{\prime}=f(x, y)$ as $h \rightarrow 0$. It is more difficult to predict the behavior of the $y_{n}$ for finite $h$ as weak instabilities may occur.

Denoting by $\zeta_{\nu}(H), \nu=1(1) k$, the zeros of the polynomial

$$
\varphi(z, H):=\rho(z)-H \sigma(z),
$$

we know from $[1$, p. 238], that for a scheme $(2)$ of order $p$ there is one zero, which we will always denote by $\zeta_{1}(H)$, which satisfies

$$
\zeta_{1}(H)=e^{H}+O\left(H^{p+1}\right) .
$$

For a given value of $H$ (real) we will call a $\mathrm{D}$-stable scheme (2) strongly stable if ${ }^{2}$

Received May 27, 1964. The research reported in this paper was supported in part by the U.S. Air Force under Grant No. AF EOAR 63-77 through the European Office, OAR.

${ }^{1}$ For Dahlquist-stable (cf. [3]).

${ }^{2}$ See Remark at the end of this section. 


$$
\left|\zeta_{\nu}(H)\right| \leqq \zeta_{1}(H), \quad \nu=2(1) k,
$$

and weakly unstable otherwise.

Each D-stable scheme is strongly stable for $H=0$, by continuity there will be a largest number $H^{+} \geqq 0$ and a smallest number $H^{-} \leqq 0$ such that (2) is strongly stable for each $H$ from the stability interval $\left[H^{-}, H^{+}\right] .^{2}$

It is evident for constant $g(x):=f_{y}(x, y(x))$ and confirmed by experience for variable $g$ that the solution $y_{n}$ of (2) simulates the behavior of $y(x)$ if $h g$ remains within the stability interval. For a weakly unstable scheme (e.g., MilneSimpson's method (1)) $H^{-}=0$ and the method should not be used for $g<0$.

If $\beta_{k} \neq 0,(2)$ defines $y_{n+k}$ implicitly and is usually replaced by the predictorcorrector scheme ${ }^{3}$

$$
\begin{aligned}
& y_{n+k}^{(0)}=-\sum_{\nu=0}^{k-1} \alpha_{\nu}{ }^{*} y_{n+\nu}+h \sum_{\nu=0}^{k-1} \beta_{\nu}{ }^{*} f_{n+\nu}, \\
& y_{n+k}^{(i)}=-\sum_{\nu=0}^{k-1} \alpha_{\nu} y_{n+\nu}+h\left(\sum_{\nu=0}^{k-1} \beta_{\nu} f_{n+\nu}+\beta_{k} f\left(x_{n+k}, y_{n+k}^{(i-1)}\right)\right), \quad i=1(1) m .
\end{aligned}
$$

A simple computation shows that for the algorithm (6) the polynomial (3) is transformed into ${ }^{4}$

$$
\varphi^{m}(z, H):=\left(1-B^{m}\right)(\rho(z)-H \sigma(z))+B^{m}(1-B)\left(\rho^{*}(z)-H \sigma^{*}(z)\right)
$$

with $B:=H \beta_{k}, \rho^{*}(z):=\sum_{\nu=0}^{k} \alpha_{\nu}{ }^{*} z^{\nu}, \alpha_{k}{ }^{*}=1, \sigma^{*}(z):=\sum_{\nu=0}^{k-1} \beta_{\nu}{ }^{*} z^{\nu}$. Obviously $\lim _{m \rightarrow \infty} \varphi^{m}(z, H)=\varphi(z, H)$ if $|B|<1$.

Assume that the predictor is of order $q \geqq 0$. It is clear from (3), (4), and (7) that the zeros $\zeta_{\nu}{ }^{m}$ of $\varphi^{m}$ satisfy (after a suitable ordering)

$$
\begin{aligned}
& \zeta_{1}{ }^{m}(H)=e^{H}+O\left(H^{p+1}\right)+O\left(H^{q+m+1}\right), \\
& \zeta_{\nu}{ }^{m}(H)=\zeta_{\nu}(H)+O\left(H^{m}\right) .
\end{aligned}
$$

For all weakly unstable schemes of practical importance the violation of $(5)$ for $H<0$ is a first-order effect in $H$, hence only the zeros $\zeta_{\nu}{ }^{1}$ of $\varphi^{1}$ may possibly not share the undesirable behavior of the $\zeta_{\nu}{ }^{5}$ Therefore we may restrict our considerations to the case $m=1$; we will-for given weakly unstable schemes-attempt to select $\left(\rho^{*}, \sigma^{*}\right)$ such that the stability interval for $\varphi^{1}$ has $H=0$ as an interior point.

Remark: Some authors (e.g., [5]) replace (5) by $\left|\zeta_{\nu}(H)\right| \leqq 1$ in the definition of a stability interval. This seems not appropriate since, e.g., a 2 -step scheme with $\zeta_{2}(H)=-1-H / 2+O\left(H^{2}\right)$ will also generate oscillations growing exponentially relative to the true solution if used for $y^{\prime}=-y$.

3. Selection of the Predictor. From now on we will only consider the polynomial $\varphi^{1}(x, H)$ and its zeros $\zeta_{\nu}{ }^{1}(H), \nu=1(1) k$, hence we will omit the superscript 1 . Furthermore we define $\zeta_{\nu 0}:=\zeta_{\nu}(0)$.

${ }^{3}$ If the predictor reaches back farther than the corrector the degree $k$ of the corrector has to be formally raised accordingly.

${ }^{4}$ This assumes a $P(E C)^{m} E$ algorithm (cf. [4]); for a $P\left(E\left(^{\prime}\right)^{m}\right.$ algorithm the situation is more complicated. See footnote 5 , however.

${ }^{5}$ Since (8) holds equally for $P(E C)^{m}$ algorithms (see [4]) our conclusion is also true for this case. 
If $\left|\zeta_{\nu 0}\right|<1$ for a certain $\nu>1,(5)$ has to hold in a full vicinity of $H=0$ by continuity. Therefore it suffices to consider $\nu \in W:=\left\{\nu: 2 \leqq \nu \leqq k,\left|\zeta_{\nu 0}\right|=1\right\}$. For $\nu \in W$, let

$$
\left|\zeta_{\nu}(H)\right|=1+A_{\nu} H+B_{\nu} H^{2}+O\left(H^{3}\right) .
$$

As $p \geqq 2$ in all cases of interest, (4) and (5) yield the following necessary condition:

$$
\begin{aligned}
& \text { (a) } A_{\nu}=1, \quad \text { for } \nu \in W \text {. } \\
& \text { (b) } B_{\nu} \leqq \frac{1}{2},
\end{aligned}
$$

If the equality is excluded in (10b), condition (10) is sufficient as well to guarantee a stability interval with $H^{-}<0, H^{+}>0$. (For $B=\frac{1}{2}$, the third order terms would have to be investigated.) To find expressions for the $A_{\nu}$ and $B_{\nu}$ we derive, from

$$
\begin{aligned}
\varphi^{1}(z, H) & =\left[\rho(z)-H\left(\sigma(z)-\beta_{k} \rho^{*}(z)\right)-H^{2} \beta_{k} \sigma^{*}(z)\right](1-B), \\
\zeta_{\nu}(H) & =\zeta_{\nu 0}+H \cdot \frac{\tau_{\nu}}{\rho_{\nu}{ }^{\prime}}+H^{2}\left[-\rho_{\nu}{ }^{\prime \prime} \tau_{\nu}{ }^{2} / 2 \rho_{\nu}{ }^{\prime}+\tau_{\nu} \tau_{\nu}{ }^{\prime}+\beta_{k} \rho_{\nu}{ }^{\prime} \sigma_{\nu}{ }^{*}\right] / \rho_{\nu}{ }^{2}+O\left(H^{3}\right),
\end{aligned}
$$

'where $\tau(z):=\sigma(z)-\beta_{k} \rho^{*}(z)$, the prime denotes differentiation, and $\rho_{\nu}:=\rho\left(\zeta_{\nu 0}\right)$, etc. $\rho_{\nu}{ }^{\prime} \neq 0$ for a D-stable scheme and $\nu \in W$. Let $\zeta_{\nu 0}=e^{i \omega_{\nu}}$, then (10a) becomes

$$
\operatorname{Re}\left\{e^{-i \omega_{\nu}} \frac{\tau_{\nu}}{\rho_{\nu}^{\prime}}\right\}=1
$$

Since $\tau_{\nu}$ is linear in the coefficients $\alpha_{\nu}{ }^{*}$ of $\rho^{*}$, for given $\rho, \sigma$, condition (10a) takes the form of a linear relation between the $\alpha_{\nu}{ }^{*}$ (which are assumed real) for each $\nu \in W$.

Condition (10b) becomes an inequality which is quadratic in the $\alpha_{\nu}{ }^{*}$ and linear in the $\beta_{\nu}{ }^{*}$ : Using (12a) we have

$$
\operatorname{Re}\left\{e^{-i \omega_{\nu}} \psi_{\nu}\right\}+\frac{1}{2}\left|\frac{\tau_{\nu}}{\rho_{\nu}{ }^{\prime}}\right|^{2} \leqq 1,
$$

where $\psi_{\nu}$ denotes the coefficient of $H^{2}$ in (11). Since the corrector must not be iterated according to our analysis, the order $q$ of the predictor must be no less than $p-1$ if the original order $p$ of the corrector is to be maintained for the predictor-corrector scheme (6) with $m=1$ (see, e.g., [1, p. $259 \mathrm{ff}$.]). The requirement of a certain order $q$ for the predictor generates $q+1$ homogeneous linear relations between the $\alpha_{\nu}{ }^{*}$ and $\beta_{\nu}{ }^{*}$. Thus the following procedure seems appropriate for the determination of a suitable $\left(\rho^{*}, \sigma^{*}\right)$ for a given weakly unstable scheme $(2)$ : Evaluate (12a) in terms of the $\alpha_{\nu}{ }^{*}$, then express $\rho^{*}$ and $\sigma^{*}$ in terms of the free parameters (if any) which are left after accounting for the order relations and (12a). Then interpret (12b) as a restriction in the space of these free parameters (or check its validity).

Remark: The same considerations can be carried through for $P(E C)^{1}$ algorithms. However, the details are more involved.

4. Application. For Milne-Simpson's 2-step scheme (1) we have $\rho=z^{2}-1$, $\sigma=\left(z^{2}+4 z+1\right) / 3, p=4$, and $\zeta_{20}=-1$. As we have to require $q=3$, it seems futile to look for a stabilizing predictor with $k=2$ since the order relations alone 
determine $\rho^{*}, \sigma^{*}$ in this case:

$$
\rho^{*}=z^{2}+4 z-5, \quad \sigma^{*}=4 z+2 .
$$

Yet by a marvelous coincidence this is a predictor which does the trick:

$$
\begin{aligned}
-1 \cdot \frac{\sigma(-1)+\beta_{2} \rho^{*}(-1)}{\rho^{\prime}(-1)} & =+1, \\
-\psi(-1)+\frac{1}{2}\left(\frac{\tau_{2}}{\rho_{2}^{\prime}}\right)^{2} & =-\frac{1}{3}<1 .
\end{aligned}
$$

Therefore the algorithm

$$
\begin{aligned}
& y_{n+2}^{(0)}=-4 y_{n+1}+5 y_{n}+2 h\left(2 f_{n+1}+f_{n}\right), \\
& y_{n+2}=y_{n}+\frac{h}{3}\left(f_{n+2}^{(0)}+4 f_{n+1}+f_{n}\right)
\end{aligned}
$$

is a genuine 2 -step method of order 4 which is strongly stable for arbitrary $H$ (as it turns out), i.e., it can be safely used for $g<0$ as well as for $g>0$. Numerical results which have been obtained with (14) are shown in Section 5.

Admitting 3-step predictors, we could at first try to achieve $q=4$ : All predictors

$$
\begin{aligned}
\rho^{*} & =z^{3}+\left(8+\alpha_{0}{ }^{*}\right) z^{2}-9 z-\alpha_{0}{ }^{*}, \\
\sigma^{*} & =\left[\left(17+\alpha_{0}{ }^{*}\right) z^{2}+\left(14+4 \alpha_{0}{ }^{*}\right) z-\left(1-\alpha_{0}{ }^{*}\right)\right] / 3
\end{aligned}
$$

are of order 4 (see, e.g., [6, p. 201]), so it seems that we have one parameter left for the satisfaction of (12). However, upon introduction of the above $\rho^{*}$ into (12a), the parameter $\alpha_{0}{ }^{*}$ drops out and the necessary condition cannot be satisfied: There is no stabilizing 3-step predictor of order 4. Among the 3 -step predictors with $q=3$ the following one-parameter family is found to be stabilizing:

$$
\begin{aligned}
& \rho^{*}=z^{3}+\left(4+\alpha_{0}{ }^{*}\right) z^{2}-5 z-\alpha_{0}{ }^{*}, \\
& \sigma^{*}=\left[\left(12+\alpha_{0}{ }^{*}\right) z^{2}+\left(6+4 \alpha_{0}{ }^{*}\right) z+\alpha_{0}{ }^{*}\right] / 3,
\end{aligned}
$$

For $\alpha_{0}{ }^{*}=0$, which is well within the stabilizing region, we recover our 2-step predictor (13). Since the error term of (15) is $h^{4} y^{\mathrm{IV}} / 6$ independently of $\alpha_{0}{ }^{*}$ there is no indication why one should not choose the simpler predictor (13) and discard the 3 -step predictors.

5. Comparison with Runge-Kutta, Numerical Results. In the case of an equation $y^{\prime}=g y, g=$ const, the relative discretization error

$$
e_{\mathrm{r}}\left(x_{n}, h\right):=\left(y_{n}(h)-y\left(x_{n}\right)\right) / y\left(x_{n}\right)
$$

will behave approximately ${ }^{6}$ like $C g^{5}\left(x-x_{0}\right) h^{4}$ with

$$
C= \begin{cases}+\frac{1}{180} & \text { for the exact solution of (1), } \\ -\frac{1}{45} & \text { for the stabilized scheme (14), } \\ -\frac{1}{120} & \text { for the classical Runge-Kutta method. }\end{cases}
$$

${ }^{6}(16)$ takes into account the first term of the asymptotic expansion of the discretization error under the assumption that the initial errors are $O\left(h^{5}\right)$. For the values of $C$, see, e.g., [1]. 
TABLE 1

Relative discretization error $e_{\mathrm{r}}(x, h)$ for $y^{\prime}=-y$

\begin{tabular}{|c|c|c|c|c|c|}
\hline \multirow[b]{2}{*}{$x$} & \multirow[b]{2}{*}{$\begin{array}{c}(14) \\
h=2^{-2}\end{array}$} & \multirow[b]{2}{*}{$\begin{array}{c}\mathrm{R} .-\mathrm{K} . \\
h=2^{-1}\end{array}$} & \multirow[b]{2}{*}{$h$} & \multicolumn{2}{|c|}{$x=10$} \\
\hline & & & & (14) & $\underset{\text { (with } 2 h \text { ) }}{\text { R.-K. }}$ \\
\hline 2 & .000244 & .001585 & $2^{-1}$ & $.0357 \quad 1363$ & .21131609 \\
\hline 4 & 493 & $\begin{array}{ll}3 & 172\end{array}$ & $2^{-2}$ & .00124629 & .00794948 \\
\hline 6 & 744 & 4762 & $2^{-3}$ & 6407 & 40130 \\
\hline 8 & 995 & 6355 & $2^{-4}$ & 377 & 2260 \\
\hline 10 & 1246 & 7949 & $2^{-5}$ & 16 & 138 \\
\hline 12 & 1498 & 9547 & $2^{-6}$ & 1 & 7 \\
\hline 14 & 1748 & 11152 & & & \\
\hline 16 & 1999 & 12786 & & & \\
\hline 18 & 2251 & 14390 & & & \\
\hline 20 & 2503 & 16002 & & & \\
\hline
\end{tabular}

TABLE 2

Relative discretization error $e_{\mathrm{r}}(x, h)$ for $y^{\prime}=-y^{2}$

\begin{tabular}{|c|c|c|c|c|c|}
\hline \multirow[b]{2}{*}{$x$} & \multirow{2}{*}{$h \stackrel{(14)}{=} 2^{-5}$} & \multirow{2}{*}{$\begin{array}{c}\text { R.-K. } \\
h=2^{-4}\end{array}$} & \multirow[b]{2}{*}{$h$} & \multicolumn{2}{|c|}{$x=10$} \\
\hline & & & & (14) & R.-K. (with $2 h$ ) \\
\hline $\begin{array}{r}5 \\
10 \\
15 \\
20\end{array}$ & $\begin{array}{l}36.7 \cdot 10^{-9} \\
20.0 \\
13.9 \\
10.6\end{array}$ & $\begin{array}{l}34.9 \cdot 10^{-9} \\
19.2 \\
13.4 \\
10.4\end{array}$ & $\begin{array}{l}2^{-1} \\
2^{-2} \\
2^{-3} \\
2^{-4} \\
2^{-5} \\
2^{-6}\end{array}$ & $\begin{array}{r}.001452234 \\
96792 \\
5657 \\
334 \\
20 \\
1\end{array}$ & $\begin{array}{rr}-.005307526 \\
+\quad 18899 \\
4237 \\
299 \\
19 \\
1\end{array}$ \\
\hline
\end{tabular}

Obviously, the stabilization of (1) has to be paid for by a loss in accuracy such that the stabilized version of (1) is less accurate than R.-K. However, basing the comparison on an equal number of evaluations of $f$ for a given interval of integration (see [4]) we find that the error of (14) is only $\frac{1}{6}$ of that for R.-K. Hence we may expect that (14) is a rather effective fourth order method for the numerical integration of ordinary differential equations.

The following differential equations were solved by the predictor-corrector scheme (14) and by R.-K.: (a) $y^{\prime}=-y$, (b) $y^{\prime}=-y^{2}$, each with $y(0)=1$, for $x \leqq 20$. The value of $y(h)$ for scheme (14) was computed by one execution of R.-K.; this introduces an error of $O\left(h^{5}\right)$.

It is clear that the usual Milne-Simpson algorithm would have failed on both equations over such a long interval. ${ }^{7}$ With algorithm (14) not the least sign of an oscillation or an undue round-off accumulation was found on either differential equation. As to be expected from (16), for eq. (a) the error with (14) was less than

${ }^{7}$ Although for eq. (b) the oscillations will grow only like $h(x+1)^{8 / 3}$ relative to the basic discretization error, this constitutes an intolerable disturbance for large $x$. 
$20 \%$ of that with R.-K. (and equal effort) throughout the interval and for all stepsizes used. Some numerical values are shown in Table 1.

For the nonlinear equation (b), the errors of (14) and R.-K. were practically equal for small stepsizes. For very large steps R.-K. was poorer, with decreasing $h$ the discretization error changed its sign and became smaller (see Table 2). (This effect is caused by the complicated error terms of R.-K. which contain various derivatives of different order.) Due to this unsystematic behavior of the discretization error Richardson-extrapolation was not applicable for $R$.-K. while it worked well for (14) where the error decreased like $h^{4}$ approximately for large and small $h$.

Acknowledgment. The preparation of this paper was stimulated through some results obtained by $A$. Neiss in a systematic experimental investigation on the effect of predictor-corrector interaction upon stability [7].

Mathematisches Institut

Technische Hochschule

München, Germany

1. P. Henrici, Discrete Variable, Methods in Ordinary Differential Equations, Wiley, New York, 1962. MR 24 \#B1772.

2. W. E. MiLne \& R. R. ReYnolds, "Stability of a numerical solution of differential equations. I \& II," J. Assoc. Comput. Mach., v. 6, 1959, p. 196-203 and v. 7, 1960, p. 46-56.

3. G. DAHLQUIST, "Convergence and stability in the numerical integration of ordinary differential equations," Math. Scand., v. 4, 1956, p. 33-53. MR 18, 338.

4. T. E. HULl \& A. L. CreEmer, "Efficiency of predictor-corrector procedures," J. Assoc. Comput. Mach., v. 10, 1963, p. 291-301. MR 27 \#367.

5. H. S. WILF, "Maximally stable numerical integration," J. Soc. Indust. Appl. Math., v. 8, 1960 , p. $537-540$.

6. R. W. Hamming, Numerical Methods for Scientists and Engineers, McGraw-Hill, New York, 1962. MR $25 \# 735$.

7. A. NeIss, Untersuchungen zur Stabilität von Predictor-Corrector-Verfahren, Diplomarbeit, Techn. Hochschule München, 1964. 